\title{
STUDIES ON THE CLUSTERED RECESSIVE LETHAL GENES ON THE SECOND CHROMOSOME OF DROSOPHILA MELANOGASTER
}

\author{
SHIGEMITSU TANO \\ Laboratory of Radiation Genetics, Faculty of Agriculture, The University of Tokyo, \\ Bunkyo-ku, Tokyo
}

Received January 31, 1966

The recessive lethal genes on the second chromosome of Drosophila melanogaster have been extensively investigated by many workers. Spontaneous recessive lethals are almost always found in the natural populations and they are also induced by the artificial methods.

A number of arguments have been presented for the distribution of the recessive lethal genes on the Drosophila chromosome. Paik (1959) suggested the non-random distribution of the recessive lethals on the second chromosome in a Korean population of Drosophila melanogaster. Spiess et al. (1963) located the spontaneous recessive lethal genes extracted from a laboratory population and showed some clustering nature on the second and the third chromosomes. But they did not conclude the non-random distribution, because their sample of the lethals were not enough, if the number of estimated possible loci of the lethal genes on the chromosome was considered.

In the present study, one lethal gene cluster was found by using an overlapping deficiency technique and analyzed more precisely for the relationship among the lethal genes in this cluster.

\section{MATERIALS AND METHODS}

One hundred and thirty two second chromosome recessive lethals which have been extracted from the wild and laboratory populations of Drosophila melanogaster or induced by irradiation were kindly supplied by many laboratories. They have been extracted from the wild population of Kentucky, Massachusetts, Ohio, Texas, Virginia, Washington in U. S. A., Jugoslavia, Korea, Samoa and Japan. Some of the lethals were obtained from X-irradiated progenies and also from laboratory populations. All second chromosomes carrying the lethal genes were balanced by $C y$ inversion. Almost of all stocks had the background chromosome constitution of the original populations.

These lethal genes were selected for the region 55.1 of the second chromosome by using overlapping deficiency technique.

Six deficiency stocks which were overlapping each other at 55.1 region from left and right with different parts and length of the deficient chromosome were available to the present experiment. 
Relationships between five among six different deficiency stocks were investigated by Morgan, Bridges and Schultz (1938) and Morgan, Schultz, Bridges and Curry (1939).

Two recessive marker genes, rolled $(r l)$ and straw $(s t w)$ were available at this region and they were separable each other by recombination in spite of the same location on the linkage map by Bridges and Brheme (1944). One deficiency stock (M(2)50 $\left.0^{\mathrm{j} 7}\right)$ fell between these two markers (Figs. 2 and 3). Distance between these two markers was measured by recombination tests as a range from 0.019 to 0.037 map units (Table 1 ). The recessive lethals in this region were located by following cross:

$$
\frac{1(2)}{\mathrm{Cy}} \times \frac{\mathrm{Df}(2)}{\mathrm{Cy}}
$$

where $\mathrm{Df}(2) / \mathrm{Cy}$ has a deficient part of the chromosome around the region 55.1. Expected progenies:

$$
\frac{1(2)}{\mathrm{Cy}} \frac{1(2)}{\mathrm{Df}(2)} \frac{\mathrm{Df}(2)}{\mathrm{Cy}} \frac{\mathrm{Cy}}{\mathrm{Cy}} \text { (non-viable) }
$$

If the deficient part of the chromosome would cover the lethal gene, $1(2) / \operatorname{Df}(2)$ individual should be non-viable, therefore, all $C y$ cultures would be expected in succeeding generation. All lethal stocks selected for the 55.1 region were tested for allelism by crossing each other. Then, it was attempted to measure the distance between these clustered nonallelic lethal genes by recombination test. Crosses were set up as follows:

Cross 1.

$$
\frac{1_{\mathrm{i}}+}{\mathrm{Cy}} \times \frac{+1_{\mathrm{j}}}{\mathrm{Cy}}
$$

Cross 2.

$$
\frac{1_{\mathrm{i}}+}{+1_{\mathrm{j}}} \times \frac{\mathrm{Df}(2)_{\mathrm{k}}}{\mathrm{Cy}}
$$

Expected progenies :

$$
\frac{1_{\mathrm{i}}+}{\operatorname{Df}(2)_{\mathrm{k}}} \frac{+1_{\mathrm{j}}}{\operatorname{Df}(2)_{\mathrm{k}}} \frac{1_{\mathrm{i}} 1_{\mathrm{j}}}{\operatorname{Df}(2)_{\mathrm{k}}} \frac{++}{\operatorname{Df}(2)_{\mathrm{k}}} \frac{1_{\mathrm{i}}+}{\mathrm{Cy}} \frac{+1_{\mathrm{j}}}{\mathrm{Cy}} \frac{1_{\mathrm{i}} 1_{\mathrm{j}}}{\mathrm{Cy}}+\frac{+}{\mathrm{Cy}}
$$

$\operatorname{Df}(2)_{\mathrm{k}}$ covers both $1_{\mathrm{i}}$ and $1_{\mathrm{j}}$, therefore, the recombinant $++/ \operatorname{Df}(2)_{\mathrm{k}}$ is only the type which shows non-Cy wing. All recombinants that showed phenotypically wild type wing, were crossed with the same virgin $\mathrm{Df}(2)_{\mathrm{k}} / \mathrm{Cy}$ females in order to know whether they were true recombinants or not. The recombination values were calculated by the frequency of the male recombinants, because the female recombinants were not virgin and no way to prove the true female recombinants by test cross at the time of progeny counting.

\section{RESULTS}

First of all, avilable deficiency stocks around the 55.1 region were tested against two recessive markers, that is rolled $(r l)$ and straw (stw), to know whether the deficient part of the chromosome does cover these two loci. Secondly, these deficiency stocks were crossed each other for all possible combinations to test the allelic relationship in 


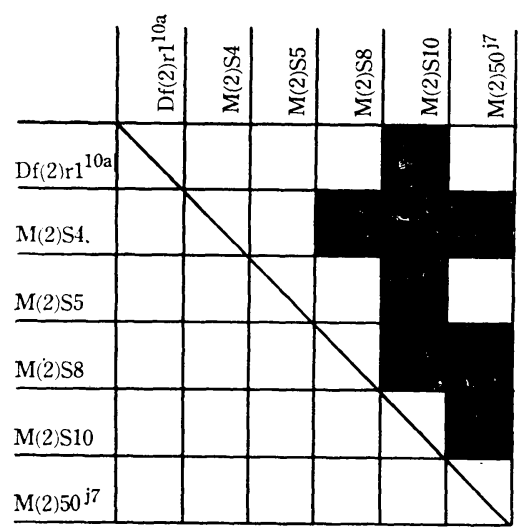

Fig. 1. Allelism tests among six deficiency stocks: open square --non-allelic; black square -allelic.

order to know the overlapping pattern. Five deficiencies, that is, $\operatorname{Df}(2) \mathrm{r}^{10 a}, \mathrm{M}(2) \mathrm{S} 4, \mathrm{M}(2) \mathrm{S} 5$, $\mathrm{M}(2) \mathrm{S} 8$, and $\mathrm{M}(2) \mathrm{S} 10$, were investigated for overlappng pattern by Morgan et al. (1938, 1939). These results are shown in Figs. 1 and 2. Another deficiency stock ( $\left.M(2) 50^{\mathrm{j} 7}\right)$ was also tested to the above mentioned five deficiencies.

Two recessive marker genes $r l$ and stw have been located by Bridges (1925) at the same position (2-55.1). Morgan et al. (1938) showed that it is possible to separate $r l$ and stw by using overlapping deficiencies as previously mentioned. Their results indicate that $r l$ is located to the left of stw. A homo-

zygous $r l$ and $s t w^{3}$ was bred from the separate stocks for the determination of the recombination distance between these two genes. Cis- and trans-phase recombination tests were performed and the results gave significantly different values. However, it was indicated that $r l$ and stw were extremely close together, perhaps about 0.03 map units (Table 1).

After establishment of the screening technique as mentioned previously, 132 spontaneous and the induced second chromosome recessive lethals, that have different origin,

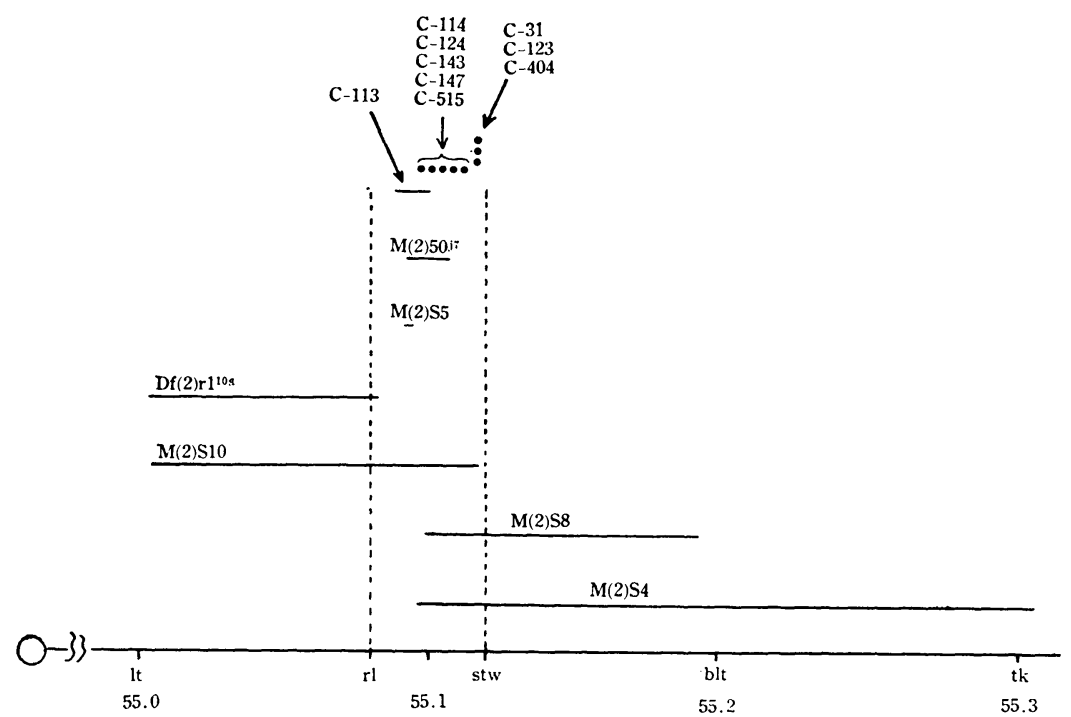

Fig. 2. Overlapping pattern of deficiencies and location of recessive lethal genes. 
Table 1. Recombination distance between rolled and straw

Cis-phase

\begin{tabular}{|c|c|c|c|c|c|c|}
\hline Expt. & $\frac{\mathrm{rl} \mathrm{stw}}{++}$ & $\frac{\mathrm{rl} \mathrm{stw}^{3}}{\mathrm{rl} \mathrm{stw}^{3}}$ & $\frac{\mathrm{rl}+}{\mathrm{rl} \mathrm{stw}}$ & $\frac{+ \text { stw }^{3}}{\text { rl stw }}$ & Total & Map Units \\
\hline 1 & 15095 & 12049 & 4 & 6 & 27154 & $0.037 \pm 0.012$ \\
\hline 2 & 9447 & 12332 & 4 & 4 & 21787 & $0.037 \pm 0.013$ \\
\hline Total & 24542 & 24381 & 8 & 10 & 48931 & $0.037 \pm 0.009$ \\
\hline \multicolumn{7}{|c|}{ Trans-phase } \\
\hline Expt. & $\frac{\mathrm{rl}+}{\mathrm{rl} \mathrm{stw}^{3}}$ & $\frac{+\mathrm{stw}^{3}}{\mathrm{rl} \mathrm{stw}^{3}}$ & 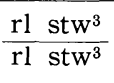 & $\frac{+\quad+}{\text { rl stw }}$ & Total & Map Units \\
\hline 1 & 9362 & 9348 & 3 & 0 & 18713 & $0.016 \pm 0.009$ \\
\hline 2 & 9795 & 10152 & 3 & 1 & 19951 & $0.020 \pm 0.010$ \\
\hline 3 & 8448 & 8827 & 1 & 3 & 17279 & $0.023 \pm 0.012$ \\
\hline 4 & 6428 & 7088 & 2 & 0 & 13518 & $0.015 \pm 0.011$ \\
\hline Total & 34033 & 35415 & 9 & 4 & 69461 & $0.019 \pm 0.005$ \\
\hline
\end{tabular}

were selected for the region 55.1 on the second chromosome. Nine out of 132 lethals (C-31, C-113, C-114, C-123, C-124, C-143, C-147, C-404 and C-515) fell between $r l$ and stw. As the results of the allelism tests of these nine lethals, almost of all combinations were non-allelic except four of them showed allelism (Fig. 3). C-113 and C-114 were allelic by lethal allelism test, however, they were separable by deficiency test (Fig. 4). C-31,

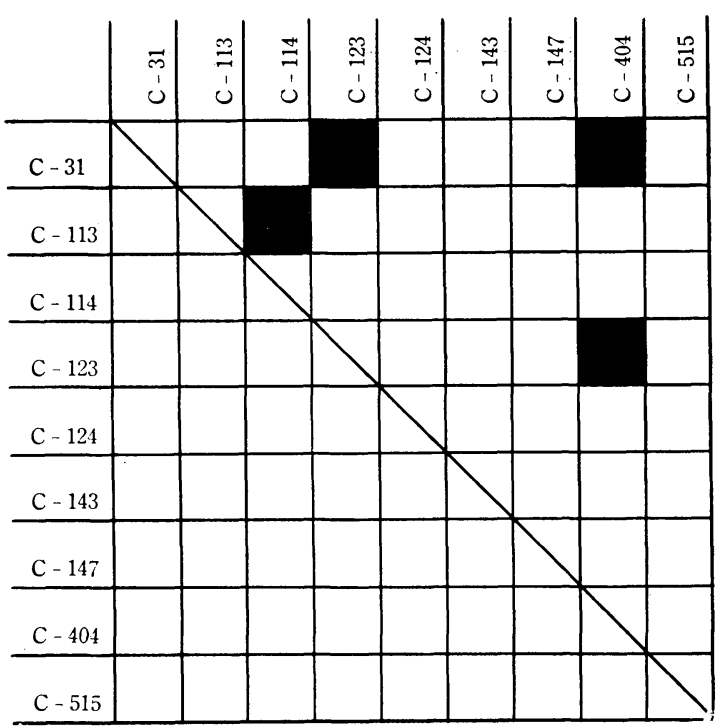

Fig. 3. Allelism tests among nine lethal genes at 55.1 on the second chromosome: open square-- nonallelic; black square-- allelic.

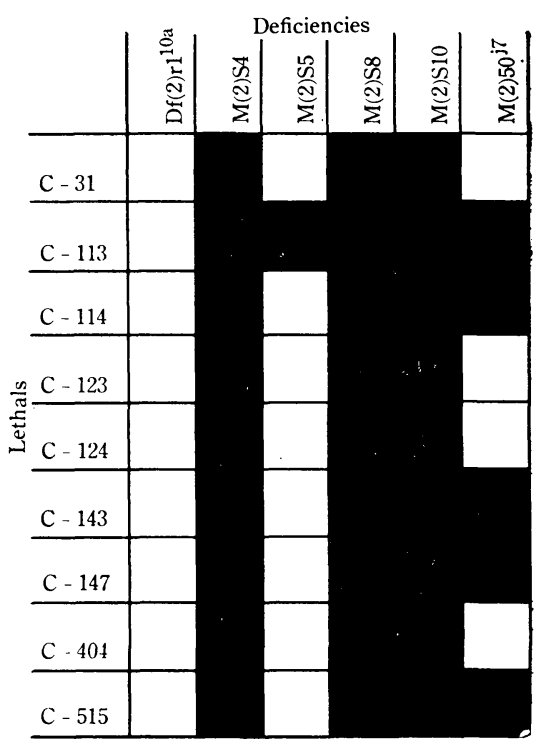

Fig. 4. Allelism tests between six deficiencies and nine recessive lethal genes: open square; non-allelic, and black; square allelic. 


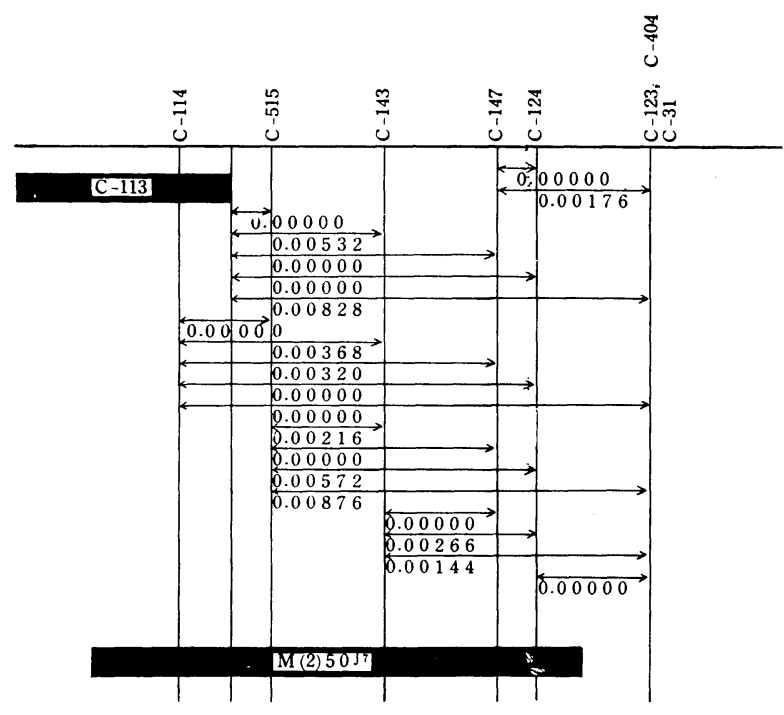

Fig. 5. Recombination map of nine recessive lethal genes at 55.1 on the second chromosome.
C-123 and C-404 were allelic each other, but these three lethal genes had different origin.

Nine recessive lethals were assumed to be located very close together, therefore, the experiment was set up in such a way to detect the distance between these clustered lethals by the recombination test. Number of experiment was different from one combination to another, therefore, the recombination values were calculated based upon the pooled data, though each data showed some inconsistent tendency in every lethal combinations. The results are shown in Table 2. Proper deficiency stock for the tester of the recombinant was employed in each experiment. Half of the male recombinants was recovered, since $\operatorname{Df}(2)_{\mathrm{k}} / 1_{\mathrm{i}} 1_{\mathrm{j}}$ was non-viable, therefore, the recombination values were obtained based upon the data including the estimated values of non-viable recombinants. Relative map distance among nine lethals are presented in Fig. 5.

Table 2. Distances between nine lethal genes at 55.1 on the second chromosome

\begin{tabular}{lccc}
\hline \hline $\begin{array}{l}\text { Lethal } \\
\text { combination }\end{array}$ & $\begin{array}{l}\text { Total no. } \\
\text { of flies }\end{array}$ & $\begin{array}{l}\text { No. of } \\
\text { recombinant }\end{array}$ & $\begin{array}{l}\text { Recombination } \\
\text { value }\end{array}$ \\
\hline $\mathrm{C}-123 \vdots \mathrm{C}-113$ & 120875 & 5 & 0.00828 \\
$\mathrm{C}-124 \vdots \mathrm{C}-113$ & 141259 & 0 & 0.00532 \\
$\mathrm{C}-143 \vdots \mathrm{C}-113$ & 150177 & 4 & \\
$\mathrm{C}-147 \vdots \mathrm{C}-113$ & 56042 & 0 & \\
$\mathrm{C}-115 \vdots \mathrm{C}-113$ & 134547 & 0 & 0.00368 \\
$\mathrm{C}-124 \vdots \mathrm{C}-114$ & 49622 & 0 & 0.00320 \\
$\mathrm{C}-143 \vdots \mathrm{C}-114$ & 46729 & 1 & 0.00876 \\
$\mathrm{C}-147 \vdots \mathrm{C}-114$ & 54349 & 1 & 0.00144 \\
$\mathrm{C}-515 \vdots \mathrm{C}-114$ & 60481 & 0 & 0.00266 \\
$\mathrm{C}-124 \vdots \mathrm{C}-123$ & 65357 & 6 & 0.00176 \\
$\mathrm{C}-143 \vdots \mathrm{C}-123$ & 136932 & 0 & \\
$\mathrm{C}-143 \vdots \mathrm{C}-124$ & 122486 & 1 & 0.00572 \\
$\mathrm{C}-147 \vdots \mathrm{C}-123$ & 139677 & 2 & 0.00216 \\
$\mathrm{C}-147 \vdots \mathrm{C}-124$ & 150804 & 1 & \\
$\mathrm{C}-515 \vdots \mathrm{C}-143$ & 113019 & 0 & \\
$\mathrm{C}-515 \vdots \mathrm{C}-143$ & 69202 & 4 & 2 \\
$\mathrm{C}-515 \vdots \mathrm{C}-147$ & 139711 & 0 & \\
\hline
\end{tabular}




\section{DISCUSSION AND CONCLUSION}

A number of studies have been concentrated on the problem of the second chromosome recessive lethals of Drosophila melanogaster, such as the mechanism of occurrence of the recessive lethal mutations and their distribution on the chromosome.

Number of the possible second chromosome recessive lethal loci has been assumed as 495 by Ives (1945) and 400 by Wallace (1950). As for the distribution of the lethal genes on the second chromosome, it has been discussed whether they distribute at random or non-random.

Nine out of 132 recessive lethal genes were located at almost the same position on the second chromosome of Drosophila melanogaster in this experiment. There were complete complementation among these lethal genes, except between $\mathrm{C}-113$ and $\mathrm{C}-114$, and also C-31, C-123 and C-404. The former was allelic each other by allelism test, however, these two lethals were separable by the two deficiency stocks, that is $\mathrm{M}(2) 50^{\mathrm{jz}}$ and M(2)S5 (cf. Fig. 4). One of the possible explanations to this relationship is that C-113 has a small deficient part of chromosome which covers the lethal gene of C-114. However, C-113 does not cover the $r l$ locus, therefore, this should be a very small deficiency. This result indicates that some of the lethals reported as a lethal gene mutation should have been classified as a deficiency, if appropriate screening technique were available. Therefore, it suggests the difficulty of detecting whether the lethals are genic or the minor chromosomal aberrations.

The latter was allelic each other and could not be separated by deficiency test, therefore, they were assmed to be the identical lethals having different origin.

Two recessive marker genes, $r l$ and $s t w$ were available at the 55.1 region on the second chromosome. The distance between these two genes was measured as a range from 0.037 to 0.019 map units by cis- and trans-phase recombination tests (cf. Table 1 ). Cis- and trans-phase tests gave significantly different values. This may be interpreted as an effect of the isogenic wild type background used in making the cis-phase heterozygotes; the cis-phase stock and the trans-phase heterozygote contain a random sample of the genetic background of the two separate stocks. The important point established here by these studies is that $r l$ and st $w$ are extremely close together, perhaps, about 0.03 of a map unit. It is a quite interesting phenomenon that the great amount of genetic differentiation, such as the clustered lethals, exists in such a small region of the second chromosome.

Seven loci including one small deficiency were separable by deficiency tests, therefore, the next experiment was designed to know whether these lethals do recombine each other or not. The recombination tests performed by crossing the trans-heterozygote of two lethal genes with a specific deficiency stock which covers both lethals at the same time. $\quad++/ \operatorname{Df}(2)$ is only surviving genotype having wild type wing out of eight possible genotypes in the progeny. Therefore, theoretically $1 / 2$ of the total recombi- 
nant could be recovered. The highest recombination value among seven lethal loci was obtained in between C-123 and C-515 (0.00786 map unit). None of the recombinant was recovered in some combination of the lethals, however, it would be possible to get a recombinant if sample size of the test were increased. Range of the recombination values was 0.00144 to 0.00876 map unit (cf. Table 2).

Relative order of distribution of the lethal genes within this cluster could be obtained, if the accurate recombination values were obtained. At the present stage of the experiment, two loci that is $\mathrm{C}-113$ and $\mathrm{C}-123$ were located at the both ends of this cluster. The locus of $\mathrm{C}-123$ was covered by $\mathrm{M}(2) \mathrm{S} 4, \mathrm{M}(2) \mathrm{S} 8$ and $\mathrm{M}(2) \mathrm{S} 10$, but not by $\mathrm{Df}(2) \mathrm{r}^{10 \mathrm{a}}, \mathrm{M}(2) \mathrm{S} 5$ and $\mathrm{M}(2) 50^{\mathrm{j} 7}$. Therefore, C-123 was located at the right side end. C-113 was covered by $\mathrm{M}(2) 50^{\mathrm{j} 7}$ and $\mathrm{M}(2) \mathrm{S} 5$ but not by $\mathrm{Df}(2) \mathrm{rl}{ }^{10 \mathrm{a}}$. Rest of the lethal genes (C-114, C-124, C-143, C-147 and C-515) were covered by $\mathrm{M}(2) 50^{\mathrm{j} 7}, \mathrm{M}(2) \mathrm{S} 10, \mathrm{M}(2) \mathrm{S} 8$ and $\mathrm{M}(2) \mathrm{S} 4$, but not by $\mathrm{M}(2) \mathrm{S} 5$ and $\mathrm{Df}(2) \mathrm{r}^{10 \mathrm{a}}$. These five lethals could be located at the middle part in the cluster and C-113 at the left side end (cf. Fig. 2). C-114 must be located to the right of $\mathrm{C}-113$, because $\mathrm{C}-114$ was the one that was allelic to $\mathrm{C}-113$. Location of the five lethal genes of the middle group could not be determined precisely yet, because the recombination analysis was not completed. Especially, C-147 was hardly recombine with the other lethals by the recmbination test. However, it was clear from the present experiment that the distance among these lethals was much smaller than the distance between $r l$ and stw (cf. Fig. 4).

It is quite interesting to consider the nature of the cluster of lethal genes based on the molecular terms at particular position on the chromosome.

The investigation of the structural and functional organization of genetic units in microorganisms has rapidly progressed in recent years (Benzer, 1959, 1961 ; Freese, 1959). This cluster of the recessive lethal genes is comparable to many complex loci investigated in Drosophila melanogaster, e. g., dumpy-, lozenge-, miniature-dusky-, Notch-, rosy-, white-locus, etc.

Freese (1959) hypothesized that if a gene is to be defined at all in molecular terms, it may be identified with a unit of function which is determined by a stretch of many nucleotide pairs of the order of $10^{3}$. Green (1961) presented a hypothesis that the Drosophila locus finds its counterpart in the cistron of the virus and bacterium. This is to say that the recombinationally indivisable unit of the higher organism is equivalent to a microbial unit which is divisable by the process of microbial recombination. Chovick et al. (1962) investigated on the rosy locus on the third chromosome of Drosophila melanogaster. According to their calculation, one map unit of Drosophila chromosome has been estimated to be $1.54 \times 10^{5}$ nucleotide pairs. Using the maximum estimate of the smallest distance thus far resolved in the present study, one emerges with an estimate of 222 nucleotide pairs as the distance separating C-143 and C-123. Maximum length of this lethal cluster using the maximum distance obtained for C-515 and C-123, indicates a value of 1349 nucleotide pairs. 
An estimation of the total length of the rosy cistron indicates a value of $11.8 \times 10^{3}$ nucleotide pairs (Chovick, 1962). Therefore, the recessive lethal gene cluster in this experiment is much smaller than the rosy cistron, even if this cluster were one cistron.

It is possible to conclude that the mechanism of recombination in higher organisms does indeed permit recombination within a cistron and that the resolving power of genetic analysis by recombination approaches to that of microbial system.

One of the many interesting problem involved here is to consider why this particular region of the second chromosome has high frequency of the lethal mutations. However, on the other hand, Mukai and Yoshikawa (personal communication) have tested 26 second chromosome recessive lethals that were not exposed to the natural selection and showed that none of them fell in this region. Paik (1959) has reported on the non-random distribution of the lethal genes in the second chromosome in a Korean natural population of Drosophila melanogaster. His conclusion showed that the distribution is not due solely to chance but is concentrated whether in the right arm or near the centromere region. Spiess et al. (1963) has investigated the distribution of the spontaneous lethals on the second and third chromosome extracted from Wallace's population and they showed some clustering loci on both chromosomes. They concluded from the experiment that it would be difficult to prove a non-random distribution of lethal loci along the length of either second or third chromosome, since the total linkage map of known lethal loci is far from complete. However, they said that it might be expected that spontaneous lethal mutations might occur more or less equally in both arms of the large autosomes. The clustering distribution which was found in the lethals in Wallace's population not simply due to their occurrence in region of low crossing over, becaue they are found in region of high crossing over as well as low crossing over.

It is very difficult to decide whether recessive lethal genes distribute at random on the second chromosome at the present stage of the experiment. In case the nonrandomness is considered, two possibilities come into consideration. First of all, region of the clustered lethal on the chromosome is highly unstable and mutable to lethal, that is similar to "hot spot" as Benzer $(1955,1959,1961)$ has reported on the mutants of rII group of T4 bacteriophage of E. coli. If significantly larger number of lethal mutations could be induced to the particular region on the chromosome, the hypothesis of "hot spot" would be accepted to Drosophila chromosome. Secondly, the lethal genes at the clustered region have higher fitness in some degree in the populations, therefore, they are kept in the natural or artificial populations. Fitness of the lethal genes in the population is greatly influenced by the genotypes of the background chromosome constitution. It is very difficult to decide that the clustering lethals are caused by either higher mutability of particular region of the chromosome or higher fitness of the lethal genes located to the clustered region.

It was concluded from the present experiment that many spontaneous and induced 
lethal genes are clustering at the 55.1 region on the second chromosome and they do recombine each other, therefore, it is possible to subdivide the lethal locus to more precise order.

\section{SUMMARY}

1) 132 second chromosome recessive lethals of Drosophila melanogaster selected for the region of 55.1 on the second chromosome by using overlapping deficiencies.

2) Nine lethal genes fell in this region. Three of them were allelic each other and one was assumed to be a small deficiency. The three allelic lethals were extracted from the different original populations.

3) Recombination analysis among these lethals was performed and maximum distance among these lethals was 0.00876 map unit. Distance between two recessive outside markers of this cluster was estimated to be 0.03 map unit based on cis- and trans-phase recombination tests.

\section{ACKNOWLEDGEMENT}

The author wishes to express his sincere gratitude to Dr. Takane Matsuo of the University of Tokyo for his encouragement and to Dr. Allan B. Burdick for his helpful suggestions and discussion.

$\mathrm{He}$ is particularly indebted to Dr. Terumi Mukai for his criticism and discussion and to the late Miss Maxi E. Krawinkel for her technical assistance.

\section{LITERATURE CITED}

Benzer, S., 1955 Fine structure of a genetic region in bacteriophage. Proc. Natl. Acad. Sci. U. S. 41: $344-354$.

Benzer, S., 1959 On the topology of the genetic fine structure. Proc. Natl. Acad. Sci. U. S. 45: 1607-1620.

Benzer, S., 1961 On the topography of the genetic fine structure. Proc. Natl. Acad. Sci. U. S. 47: 403-415.

Bridges, C. B., and K.S. Brheme, 1944 The mutants of Drosophila melanogaster. Carnegie lnst. Wash. Publ. No. 552. Washington D. C.

Chovick, A., A. Schalet, R. P. Kernaghan, and J. Talsma, 1962 The resolving power of genetic fine structure analysis in higher organisms as exemplified by Drosophila. Am. Naturalist 96: 281296.

Freese, E., 1959 On the molecular explanation of spontaneous and induced mutations. Brookhaven Symp. Biology 12: 63-75.

Green, M. M., 1961 Phenogenetic of the lozenge loci in Drosophila melanogaster. II. Genetics of lozenge-Kriveshenko $\left(1 z^{\mathrm{k}}\right)$. Genetics 46 : 1169-1176.

Ives, P. T., 1945 The genetic structure of American populations of Drosophila melanogaster. Genetics 30: 167-196. 
Morgan, T. H., C.B. Bridges, and J. Schultz, 1938 Constitution of the germinal material in relation to heredity. Carnegie Inst. Wash. Year Book 37: 304-309.

Morgan, T. H., J. Schultz, C. B. Bridges, and V. Curry, 1939 Investigation on the constitution of the germinal material in relation to heredity. Carnegie Inst. Wash. Year Book 38: 273-277.

Paik, Y. K., 1959 Non-random distribution of lethal genes in the second chromosome of Drosophila melanogaster in natural populations. Drosophila Inform. Serv. 33: 153.

Spiess, E. B., R. B. Helling, and M. R. Capenos, 1963 Linkage of autosomal lethals from a laboratoty population of Drosophila melanogaster. Genetics 48: 1377-1388.

Wallace, B., 1950 Allelism of second chromosome lethals in Drosophila melanogaster. Proc. Natl. Acad. Sci. U. S. 36: $654-657$. 